\title{
Utilization of the chromogenic agar MRSA ID in the surveillance of methicillin-resistant Staphylococcus aureus in a low endemic unit for MRSA
}

\author{
Laura Squarzon', Andrea Tessari', Silvia Paccagnella', Marino Scarin', Marina Munari², \\ Antonietta Cavallaro' \\ 'Microbiology and Virology, Hospital of Padua \\ ${ }^{2}$ Neurosurgery Intensive Care Unit, Hospital of Padua
}

Utilization of the chromogenic agar MRSA ID in the surveillance of methicillin-resistant Staphylococcus aureus in a low endemic unit for MRSA

Key words: MRSA, Nosocomial infection, Prevention

\section{SUMMARY}

Methicillin-resistant Staphylococcus aureus (MRSA) is one of the most common pathogen responsible for nosocomial infections. Approximately $20 \%$ of patients undergoing surgical operations acquires at least one nosocomial infection. Nasal carriers of S. aureus have been identified as one of the populations at major risk to develop this type of infection, particularly, after operations, dialysis, transplants and admission in intensive care unit. Our study was conducted on patients who came from a Neurosurgery Intensive Care Unit, where the incidence of MRSA is very low (8\%) and where it is very important to identify MRSA carriers in order to adopt measures to avoid transmission. The use of chromogenic agar MRSA ID allowed us to identify green colonies of MRSA within 24 hours and to make a timely and careful diagnosis.

Received January 7, 2008

Accepted January 30, 2008

\section{INTRODUCTION}

Methicillin-resistant Staphylococcus aureus (MRSA) was isolated for the first time in 1961 (1) and today it is among the most common pathogen responsible for nosocomial infections $(4,5)$.

Approximately $20 \%$ of all patients undergoing surgery acquires at least one nosocomial infection (15, $10)$. It has been estimated that nasal carriers of $S$. aureus are most at risk to develop nosocomial infections, especially if seropositives, cirrhotic, dialysised (haemo- and peritoneal dialysis), liver grafted hospitalized in surgery and intensive care units $(14,8)$. Infected and colonized patients are the reservoir of MRSA, and the main way of transmission from patient to patient is represented by health care workers. It is a general consensus that the faster is the diagnosis and the report of susceptibility results for MRSA, more the therapy is appropriate and the necessary control measures to prevent the spread of colonization can be established.

When compared with patients infected with methicillin-sensitive $S$. aureus (MSSA), patients with MRSA tend to develop more severe clinical conditions, to require longer periods of hospitalization, to endure more aggressive antibiotic therapy and to have more adverse prognostic factors $(9,12)$. Considering these factors, MRSA infections compared to MSSA infections lead to a significantly higher rate of mortality, morbidity, length of hospitalization and cost of treatment $(2,7,11)$.

Laboratory diagnosis and assays of antimicrobial susceptibility are milestones in controlling and preventing infection by MRSA, because they affect the procedures used in patient management and the choice of prophylaxis to follow.

The study was conducted within two months (April-June 2007) on 25 patients hospitalised in the Neurosurgery Intensive Care Unit of the Padua Hospital. This unit was chosen for its low incidence of MRSA (8\%) (3).

Three pharyngeal swabs (PS) and three nasal swabs (NS) were performed for each patient at the entry (time 0 ), the third day (time 1) and the seventh day after hospitalization (time 2), in order to monitor the eventual colonization by MRSA. The samples were seeded both on blood agar (BA) and on chromogenic agar MRSA ID (bioMérieux, Marcy l'Etoile, France) containing $4 \mathrm{mg} / \mathrm{L}$ of 
cefoxitin, an antimicrobial with proven superiority compared to other compounds, such as oxacillin, methicillin and ciprofloxacin, in the selection of MRSA against other strains of $S$. aureus (6). Two readings were carried out: 24 and 48 hours after seeding.

Direct identification of MRSA strains using the chromogenic agar supplemented with cefoxitin, which inhibits the growth of most bacteria, is based on appearance of spontaneous green colonies producing $\alpha$-glucosidase, after incubation of $18-24$ hours at $37^{\circ} \mathrm{C}$ without adding additional reagents.

In order to establish the real discriminatory capacity and the reliability of MRSA ID, the identification and the antimicrobial susceptibility test by Vitek2 (bioMérieux) was done on all colonies grown in chromogenic agar and on all catalase positive cocci grown in BA. The test of cefoxitin was also conducted, following the CLSI 2007 guidelines (13) by seeding the samples on Mueller Hinton agar plus cefoxitin $30 \mu \mathrm{g}$, incubation at $30^{\circ} \mathrm{C}$ for 24 hours.

The analysis of 150 samples revealed a total of 40 swabs positive for Staphylococcus aureus, 6 strains of which characterised by a MRSA phenotype. In all cases this phenotype was confirmed by the cultural reference method and by the antimicrobial susceptibility test, showing $100 \%$ of sensitivity and specificity of the chromogenic agar (see table 1). However, colonies of different colours other than green were found and they belonged to different microrganisms, such as coagulase-negative staphylococci or MSSA.

Table I. Comparison between traditional method and chromogenic agar for identification of MRSA from 150 samples of pathologic material

\begin{tabular}{lcc}
\hline Methods & Positive $(n)$ & Negative $(n)$ \\
\hline MRSA ID bioMeriéux & 6 & I44 \\
\hline Traditional method & 6 & I44
\end{tabular}

The 6 MRSA strains were isolated from 2 patients. In the first patient, MRSA was isolated from the nose at the hospital admission (time 0) and from the pharynx after seven days (time 2), while the samples of second patient, (PS and NS) were found to be positive after seven days of hospitalization (time 2) (see table 2). The patient who had positive samples after seven days had been previously hospitalised in another hospital with a diagnosis of polytraumatism (open wounds), causing a surgery intervention (11 days before our first sample) and antibiotic therapy. The patient who was found to be colonized at the first time was directly admitted to the Neurosurgery Intensive Care with a diagnosis of brain haemor- rhage, and subsequently subjected to surgery. The patient, however, had never been hospitalized in the previous 3 months or had been subjected to antibiotic therapy in the previous 7 days. Instead, being immunocompromised, he presented an already complicated general conditions at the admission. The use of the invasive devices (central venous, arteriosus and bladder catheters) was started only 2 hours before the first sample. In both cases, the chromogenic agar MRSA ID allowed to highlight green colonies after 24 hours, enabling rapid identification of methicillinresistant Staphylococcus aureus.

Table 2. MRSA isolation from patients' samples, at different times

\begin{tabular}{ccc}
\hline Patient MRSA positive & Sample type & Sample Timing \\
\hline I & PS & 2 \\
\hline & NS & 0 \\
\hline 2 & PS & 2 \\
\hline & NS & 2
\end{tabular}

PS, pharyngeal swab; NS, nasal swab. Sample timing: 0, moment of hospitalization; I, after three days; 2, after 7 days.

Present findings show that all patients hospitalized in intensive care units should be screened, whether they are at first hospitalization or already hospitalized, whether they are immunocompromised or not. Limiting control towards some patients from health care settings, other hospitals, with injuries, etc... could limit its effectiveness. Timing used for the sampling was appropriate, since it showed that the isolation of MRSA may happen either at the entry or after several days of hospitalization. The chromogenic agar MRSA ID, compared to the gold standard method, showed also a 100\% sensitivity and specificity. The employment of this agar was helpful for the timely and accurate diagnosis of MRSA directly from clinical samples. This could certainly make possible an immediate intervention by the health care workers in all operating units, in order to confine any colonized patients and to limit the spread of infection. This could also have a significant impact on timing of hospitalization, mortality rates, morbidity and costs of hospitalization and could also allow immediately proper antibiotic therapy.

\section{BIBLIOGRAPHY}

1. Barber M. Methicillin-resistant staphylococci. J Clin Pathol 1961; 14: 385-93.

2. Centers for Disease Control and Prevention. Communityassociated methicillin-resistant Staphylococcus aureus infections in Pacific Islanders-Hawaii, 2001-2003. MMWR Morb Mortal Wkly Rep 2004; 53: 767-70.

3. Epidemiological data provided by Microbiology and Virology, Hospital of Padua (May 2006-May 2007).

4. Diekema DJ, et al. Genetic relatedness of multidrug- 
resistant, methicillin (oxacillin)-resistant Staphylococcus aureus bloodstream isolates from SENTRY Antimicrobial Resistance Surveillance Centers worldwide, 1998. Microb Drug Resist 2000; 6: 213-21.

5. European Antimicrobial Resistance Surveillance System (EARSS). Annual Report EARSS-2003. Bilthoven, The Netherlands: RIVM, 2004.

6. Felten A, et al. Evaluation of three techniques for detection of low-level methicillin-resistant Staphylococcus aureus (MRSA): a disk diffusion method with cefoxitin and moxalactam, the Vitek 2 system, and the MRSAscreen latex agglutination test. J Clinical Microbiol 2002; 8: 2766-71.

7. French GL. Bactericidal agents in the treatment of MRSA infections-the potential role of daptomycin. J Antimicrob Chemother 2006; 58: 1107-17.

8. Garrouste-Orgeas M, et al. Colonization with methicillin-resistant Staphylococcus aureus in ICU patients: morbidity, mortality, and glycopeptide use. Infect Control Hosp Epidemiol 2001; 22: 687-92.

9. Graffunder EM, et al. Risk factors associated with nosocomial methicillin-resistant Staphylococcus aureus (MRSA) infection including previous use of antimicrobials. J. Antimicrob Chemother 2002; 49: 999-1005.
10. Kirkland KB, et al. The impact of surgical-site infections in the 1990s: attributable mortality, excess length of hospitalization, and extra costs. Infect Control Hosp Epidemiol, 1999; 20: 725-30.

11. Kowalsky TJ, et al. Epidemiology, treatment and prevention of community-acquired methicillin-resistant Staphylococcus aureus infections. Mayo Clin Proc 2005; 80: 1201-7.

12. Monnet DL. Methicillin-resistant Staphylococcus aureus and its relationship to antimicrobial use: possible implications for control. Infect Control Hosp Epidemiol 1988; 19: 552-9.

13. National Committee for Clinical Laboratory Standards. 2007. Document M100-S17, 7th ed., vol. 27, National Committee for Clinical Laboratory Standards, Wayne, Pa.

14. Pujol M, et al., Nosocomial Staphylococcus aureus bacteremia among nasal carriers of methicillin-resistant and methicillin-susceptible strains, Am J Med 1996; 100: 509-16.

15. Wisplinghoff $\mathrm{H}$, et al. Nosocomial bloodstream infections in US hospitals: analysis of 24,179 cases from a prospective nationwide surveillance study. Clin Infect Dis 2004; 39: 309-17. 